\title{
Endoscopic Carpal Tunnel Release - A Prospective Study of Functional Outcome
}

\author{
T Ravindran, MS Ortho, T Sara, FRCS, YC Loh, FRCS \\ Department of Orthopaedics, University Malaya Medical Centre, Kuala Lumpur, Malaysia
}

\begin{abstract}
To evaluate the functional outcome of patients who underwent endoscopic carpal tunnel release (ECTR). This was a prospective study in 24 patients with idiopathic carpal tunnel syndrome undergoing ECTR. Patient assessment entailing both subjective and objective tests at intervals of two and six weeks, three, six and nine months and finally at one year postoperatively. Our assessment results showed overall improvement in symptoms. As early as two weeks post-operatively, there was a low incidence of pillar pain and scar tenderness, allowing patients to return to work early. There were no serious complications. ECTR can be effectively performed with a low perioperative and late postoperative rate of complications. This minimally invasive method can be an option of surgical treatment for patients with carpal tunnel syndrome, who intend to return to work soon after surgery.
\end{abstract}

Key Words:

Carpal tunnel syndrome, Endoscopic carpal tunnel release, Pillar pain, Grip strength, Chow's technique

\section{INTRODUCTION}

Since the first carpal tunnel surgery performed in 1924 by Herbert Galloway, numerous advances have been made to refine this procedure. In general, carpal tunnel release can be performed using open or endoscopic procedures. The classic open carpal tunnel release technique involves complete division of the transverse carpal ligament and the deep fascia of the forearm under direct visualization. Most surgeons prefer the open technique due to its lower level of difficulty and shorter operative time. In the majority of patients, open release techniques lead to symptomatic relief with low complication rate. However, scar tenderness and grip weakness may occur after open release.

In an attempt to reduce postoperative morbidity, endoscopic carpal tunnel release (ECTR) was developed in late 1980s. The Agee single portal and Chow two portal carpal tunnel release utilize smaller incisions and require less dissection of the subcutaneous tissue and structures overlying the transverse carpal ligament. Further, with endoscopic release techniques, there may be less scar tenderness. Proponents suggest an earlier return to work and activities of daily living compared to the open procedure. A prospective randomized, multicentre trial comparing endoscopic versus open release showed that the endoscopic group had a more rapid improvement in strength and scar tenderness than the open group in the first three months after surgery. However, at one-year follow-up, there was no difference in patient satisfaction between the endoscopic and open release group'.

The purpose of our study is to evaluate the functional outcome of ECTR in terms of early return to work and also to verify the reproducibility and effectiveness of the modified two portal technique at our centre.

\section{MATERIALS AND METHODS}

A prospective study was carried out on 30 hands (30 cases) in 24 patients with carpal tunnel syndrome, who underwent endoscopic carpal tunnel release (ECTR) between March 2004 and July 2004 by a single surgeon (YC Loh), following failed conservative management with a wrist splint for a minimum of 3 months.

The inclusion criteria for ECTR study were patients with the clinical diagnosis of idiopathic carpal tunnel syndrome, confirmed by electrophysiological testing / nerve conduction study (NCS). The clinical diagnosis was made by assessing the symptoms of pain, tingling or numbness in the affected hand or hands (bilateral) either at night, during certain activities or at all times, the presence of Phalen test, direct compression test or Tinel sign.

Patients with recurrent carpal tunnel syndrome treated previously with surgery, patients with rheumatoid arthritis, carpal tunnel syndrome occurring during pregnancy, double crush phenomenon and malunited distal end radial fracture were excluded from the study. Dorsiflexion of the wrist of at least 20 to 30 degrees is essential for correct anatomic placement of the instruments, so patients with limited wrist extension were excluded as this surgical technique is contraindicated in such circumstances.. 


\section{Surgical Technique}

The ECTR was carried out under either regional or general anaesthesia as a day surgery. Regional anaesthesia included a brachial plexus block and Bier's block. Patients who underwent simultaneous bilateral endoscopic carpal tunnel release required general anaesthesia. With tourniquet at the upper arm, the modified two-portal Chow's technique was carried out using a single retrograde knife for the division of the transverse carpal ligament. The procedure was performed using a 4mm, 30-degree inverse endoscope.

Both proximal entry and distal exit portals were appropriately marked (Figure 1). To create the proximal portal, the extrabursal approach was used to place the slotted cannula/ ridged trochar assembly in a subligamentous position without disturbing the flexor tendons, Guyon's canal and its contents, or the median nerve. The entry portal was ulnar to the median nerve, radial to the ulnar neurovascular bundle, palmar to the flexor tendons and nearly always at the proximal wrist flexion crease, when present. The pisiform was used as a reference and its palmar palpable tip is circled for reference. A line was then drawn from the proximal tip of the pisiform radially, perpendicular to the long axis of the forearm, approximately $15 \mathrm{~mm}$ in length. A second line, approximately about $5 \mathrm{~mm}$ long, was drawn perpendicular and proximal from the end of the first line. From its proximal terminus, a line perpendicular to the second line and perpendicular to the long axis of the forearm was drawn in a radial direction, approximately $5 \mathrm{~mm}$ in length. This was the line of the surgical insertion for the entry portal and lies just ulnar to the palmaris longus tendon or median nerve. The final position of the entry portal was in line with the radial border of the fourth ray digit. The exit portal position can be established by two different methods. First, the relation to the fourth ray and its projected line on the palm was noted, then a line was drawn from the third web space perpendicularly to a line along the distal margin of the fully abducted thumb. At the intersection of these two lines, a bisection of this 90degree angle was drawn with a straight line and projected proximally and ulnarly for $1 \mathrm{~cm}$ from the vertex of these lines. The exit portal incision $(5-8 \mathrm{~mm})$ was made at this point in the palm. Alternatively, a small incision was be made in the palm at the palpable tip of the trochar, and a dissection of the nerve, artery and transverse carpal ligament was made to ensure proper exit portal placement. The target area for safe exit portal creation laid close to the distal edge of the transverse carpal ligament and was small. This exit portal was pre-planned and then confirmed by endoscope tip placement.

After a $5 \mathrm{~mm}$ transverse skin incision was made for the proximal entry portal, the forearm flexor fascia was incised longitudinally to allow introduction of the trochar introducer into the carpal tunnel. The introducer was moved side to side under the flexor retinaculum to sweep away the surrounding synovium and flexor tendons as it was being inserted distally.
The sensation of sweeping the "wash board "was felt as the introducer was swept side to side under the flexor retinaculum. The distal tip of the introducer was used to locate the distal edge of the flexor retinaculum to exit through the distal portal. The slotted trochar was then reinserted with the introducer to exit through the distal portal. The overlying flexor retinaculum was incised with a retrograde knife utilizing the inverse endoscope (Figure 2). The completion of division of the flexor retinaculum was confirmed upon sighting the overlying subcutaneous fat . The portal sites were closed with two simple absorbable sutures (Vicryl rapide 4/0). A simple dressing, wool and crepe bandage was applied to the operated hand. Early finger movement was encouraged. The patient was discharged with simple analgesics and advised not to attempt any heavy lifting with the operated hand for a period of three weeks. For the cases in this study, the minimum operative time was 10 minutes and the maximum operative time was 30 minutes (mean, 15.8 minutes).

Demographic data such as age, sex, occupation, hand dominance, hand laterality and duration of symptoms were recorded. Positive physical signs including the presence of Compression test at the carpal tunnel, positive Tinel sign, positive Phalen sign and results of nerve conduction studies were also recorded.

Both preoperative and postoperative objective assessments of grip strength, two-point discrimination, pinch strength, range of motion of the wrist joint (dorsiflexion, palmar flexion, ulnar deviation and radial deviation) and visual analogue score for pain intensity were carried out independently by our hand therapist. Postoperative pillar pain, scar tenderness, presence of Tinel sign and Phalen sign, resolution of symptoms, patient's satisfaction and return to work status were also evaluated. Housewives and retired patients were asked when they returned to their preoperative activities. All patients were assessed at two weeks, six weeks, three months, six months, nine months and one year postoperatively.

Grip and pinch strength measurements were taken in the manner recommended by the American Society of Hand Therapists. A Jamar dynamometer was used to measure the grip strength, and a pinch meter was used to measure the pinch grip. The mean of three attempts of grip and pinch strength were recorded. The range of motion of the wrist joint was measured using a goniometer. The static two point discrimination test (static 2PD ) using a MacKinnon-Dellon Disk Criminator was performed on the index finger tip. The static 2PD reading between 0 and $5 \mathrm{~mm}$ is considered normal in the hand.

A recurrence was considered to occur if the patient who had initially improved after surgery subsequently noted a return of his/her symptoms. Failure of ECTR was defined as when 
there was no improvement of symptoms after surgery. Patients with moderate symptoms (i.e., occasional pain and tingling but not pain at night) after ECTR were classified as having unsatisfactory procedures. Following surgery, those patients who reported complete relief from their symptoms and return of normal hand function were classified as having complete success from the procedure. If the patient indicated that the majority of his or her complaints were relieved following the surgery but still reported slight residual tingling over the fingers, the patient was considered to have had satisfactory results from the procedure.

Preoperative and postoperative differences and interpretation of the variables were analyzed using paired -samples t-test. A 'p' value of less than 0.05 (95\% confidence interval) was considered statistically significant.

\section{RESULTS}

The majority of the patients were women (21 out of 24 patients). The patients were mostly full-time housewives (7 patients) or in the labour sector (7 patients). Five patients had sedentary jobs, mainly office work and 5 patients were not working. The age ranged from 35 to 74 years with a mean of 54.2 years. All patients were right hand dominant and 18 out of the 30 cases involved the right hand. The majority of cases (13) had symptoms of carpal tunnel syndrome for approximately one to two years. The duration of symptoms ranged from 5 to 36 months (mean, 17.4 months). Eighteen patients $(75 \%)$ underwent unilateral ECTR and six patients (25\%) underwent simultaneous bilateral ECTR. Two patients (three cases) were excluded from the final objective data analysis as they defaulted follow-up, but were included in the subjective data by interviewing them over the phone. One patient defaulted after six weeks and the other (bilateral ECTR) defaulted after two weeks.

Preoperatively, out of 30 cases operated, nine cases (30\%) had wasting and 21 cases (70\%) had no obvious wasting of the thenar muscle. Nerve conduction studies of the median nerve showed motor latency was normal in 4 cases, mild in 9 cases, moderate in 13 cases and severe in 4 cases. Median sensory latency was normal in three cases, seven cases showed no response, nine cases were mild, nine cases were moderate and two were severe. Based on the median motor and sensory latency including conduction velocity, five cases were classified as mild, 14 moderate and 11 cases were severe.

Pre-operatively, twenty-seven cases (90\%) had positive Phalen test and 26 cases $(86.7 \%)$ had positive Tinel sign. Postoperatively at 2 weeks, only 3 cases had positive Tinel sign and Phalen test respectively (Table I \& Table II). Using the non-parametric McNemar test, these improvements were statistically significant ( $p$ value $<0.001$ ). There was an initial reduction in pinch strength at two weeks, followed by a significant improvement from six months onwards. There was also an initial reduction in grip strength at two weeks and six weeks, followed by a significant improvement from three months onwards (Table III). Similarly, there was significant improvement in all aspects of wrist motion from the postoperative sixth week onward.

At two weeks post-operative, for all cases, there was a statistically significant improvement in two-point discrimination. There was also significant improvement (i.e., relief of symptoms) according to the visual analogue scale (Table IV). Twenty-six cases (80\%) had no pillar pain, and 28 cases $(93.3 \%)$ had no scar tenderness whereas 2 cases $(6.7 \%)$ had mild scar tenderness. Twenty-four cases $(80 \%)$ reported a complete resolution and improvement of paraesthesia and six cases $(20 \%)$ reported no improvement.

At the one year postoperatively, 27 cases $(90 \%)$ showed improvement or were symptom free, two cases $(6.7 \%)$ still had numbness over the median nerve distribution similar to the preoperative level and one case $(3.3 \%)$ had numbness over the fingers, which was slightly less than the preoperative level. The two cases $(6.7 \%)$ had initial improvement of symptoms at two weeks, but subsequently had relapse of symptoms from six weeks onwards. A repeat NCS was performed. In one patient, the distal motor latency was $7.0 \mathrm{~ms}$ and the median sensory action potential was absent; open carpal tunnel release was performed at the end of one year and this patient's symptoms later improved. In the other patient, the median sensory latency was $3.4 \mathrm{~ms}$, the conduction velocity was reduced $(32.4 \mathrm{~m} / \mathrm{s})$, and motor latency was slightly prolonged to $4.65 \mathrm{~m} / \mathrm{s}$. The patient's symptoms also improved after undergoing open carpal tunnel release. An additional case (3.3\%) complained of moderate symptoms, numbness over the fingers (median nerve distribution) and occasional paraesthesia but no pain postoperatively. The repeat NCS showed a prolonged motor latency $(6.05 \mathrm{~ms})$ with slightly reduced velocity and normal compound motor action potential (CMAP). Sensory latency was prolonged to $4.45 \mathrm{~m} / \mathrm{s}$ and the sensory conduction velocity was also reduced $(24.7 \mathrm{~m} / \mathrm{s})$. The patient was advised to undergo open carpal tunnel release but did not undergo the procedure as her symptoms improved after 2 months of using a wrist splint.

All 30 cases were interviewed at the end of one year. Twentytwo cases $(73.3 \%)$ were considered a complete success, five cases $(16.7 \%)$ had satisfactory results, one case $(3.3 \%)$ was considered unsatisfactory and there were two cases $(6.7 \%)$ who had recurrence. The time to return to work for the 24 patients ranged from 7 to 35 days (mean, 15 days). There was no neurovascular injury in this study. No injury of the palmar cutaneous branch of the median nerve, the most frequent complication of the open technique ${ }^{2}$, was seen. There was no post-operative infection or reflex sympathetic dystrophy. 
Table l: Distribution of the Phalen test results preoperatively and two weeks post-operatively.

\begin{tabular}{|lcc|}
\hline Preoperative Phalen test & Negative & Post-operative Phalen test \\
& 3 cases & Positive \\
\hline 3 cases with negative tests & 24 cases & none \\
27 cases with positive tests & 3 cases \\
\hline
\end{tabular}

Table Il: Distribution of Tinel's sign results preoperatively and two weeks postoperatively.

\begin{tabular}{|lcc|}
\hline Preoperative Tinel test & Negative & Post-operative Tinel test \\
& 4 cases & Positive \\
\hline 4 cases with negative tests & 23 cases & none \\
26 cases with positive tests & 3 cases \\
\hline
\end{tabular}

Table III: Pinch strength and grip strength of study subjects.

\begin{tabular}{|c|c|c|c|c|c|c|}
\hline & \multicolumn{6}{|c|}{ Duration of follow up after surgery } \\
\hline & $\begin{array}{c}2 \text { weeks } \\
(n=30)\end{array}$ & $\begin{array}{c}6 \text { weeks } \\
(\mathrm{n}=28)\end{array}$ & $\begin{array}{c}3 \text { months } \\
(\mathrm{n}=27)\end{array}$ & $\begin{array}{c}6 \text { months } \\
(\mathrm{n}=27)\end{array}$ & $\begin{array}{c}9 \text { months } \\
(\mathrm{n}=27)\end{array}$ & $\begin{array}{l}1 \text { year } \\
(n=27)\end{array}$ \\
\hline Preoperative mean pinch $(\mathrm{kg})$ & 5.40 & 5.31 & 5.06 & 5.06 & 5.06 & 5.06 \\
\hline Postoperative mean pinch $(\mathrm{kg})$ & 4.50 & 5.15 & 5.23 & 5.57 & 5.77 & 5.90 \\
\hline Preoperative mean grip strength $(\mathrm{kg})$ & 17.30 & 17.07 & 16.26 & 16.26 & 16.26 & 16.26 \\
\hline Postoperative mean grip strength $(\mathrm{kg})$ & 9.94 & 14.05 & 17.81 & 18.09 & 19.24 & 20.50 \\
\hline
\end{tabular}

Table IV: Two point discrimination (2PD) and visual analogue scale (VAS) score for study subjects.

\begin{tabular}{|c|c|c|c|c|c|c|}
\hline & \multicolumn{6}{|c|}{ Duration of follow up after surgery } \\
\hline & $\begin{array}{c}2 \text { weeks } \\
(n=30)\end{array}$ & $\begin{array}{c}6 \text { weeks } \\
(n=28)\end{array}$ & $\begin{array}{l}3 \text { months } \\
(n=27)\end{array}$ & $\begin{array}{c}6 \text { months } \\
(n=27)\end{array}$ & $\begin{array}{l}9 \text { months } \\
(n=27)\end{array}$ & $\begin{array}{l}1 \text { year } \\
(n=27)\end{array}$ \\
\hline Preoperative mean 2 PD $(\mathrm{mm})$ & 5.0 & 5.1 & 5.0 & 5.0 & 5.0 & 5.0 \\
\hline Postoperative mean 2 PD (mm) & 3.3 & 2.9 & 2.7 & 2.8 & 2.6 & 2.6 \\
\hline Preoperative mean VAS & 6.4 & 6.3 & 6.4 & 6.4 & 6.4 & 6.4 \\
\hline Postoperative mean VAS & 4.1 & 3.6 & 2.9 & 2.4 & 2.0 & 1.7 \\
\hline
\end{tabular}

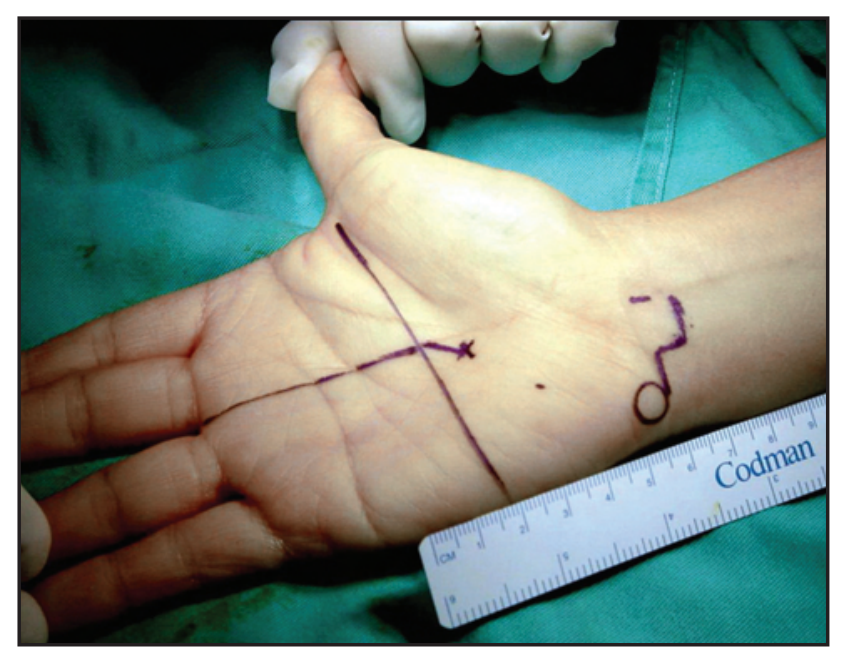

Fig. 1: Photograph showing the marking of the proximal (entry) portal and the exit portal.

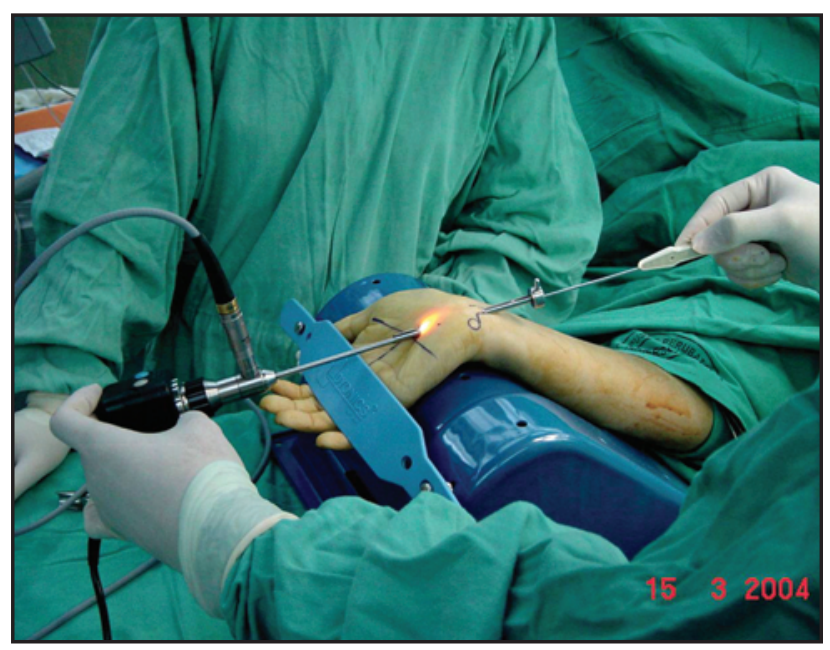

Fig. 2: Photograph showing endoscope placement in the exit portal and the retrograde (hook) knife placed through the proximal portal to release the transverse carpal ligament. 


\section{DISCUSSION}

Endoscopic carpal tunnel release (ECTR) was introduced in the late 1980s as a new technique for surgical treatment of carpal tunnel syndrome (CTS). Since then many studies have shown a high success rate for the procedure. Major controversy has ensued in the literature, however, regarding the safety, success, and, most importantly, the complication rate of this procedure. Comparative clinical studies have shown that endoscopic carpal tunnel release resulted in less postoperative pain, faster recovery of grip and pinch strength, and earlier return to work compared to open methods ${ }^{3-4}$. Palmer DH et al. ${ }^{5}$ reported a single centre prospective study that compared three different treatment methods: standard open release, single-portal endoscopic technique (Agee), and the two-portal endoscopic technique (Chow). Two hundred eleven cases in 163 patients were evaluated using a clinical outcomes questionnaire and objective testing over a 6-month follow-up period. Patients treated with open release reported more thumb weakness and pain with activities of daily living after surgery. Endoscopically treated patients achieved faster recovery of grip and pinch strength and wrist range of motion, had less mid-palm tenderness compared to the open release group and also returned to work sooner.

After surgery the majority of the patients in our study required minimal oral analgesics and were able to move the wrist joint immediately. Since ECTR is minimally invasive, it permits early return to normal activities and work. Similar to the present study in which the time to return to work for the patients ranged from 7 to 35 days (mean, 15 days), Nagle et al. reported that $81 \%$ of the non-worker's compensation patients returned to work less than 4 weeks after the procedure ${ }^{6}$. Kerr et al. found that patients treated endoscopically irrespective of insurance class, returned to work 10.6 days sooner than did those treated by open method $^{4}$.

The overall success rate in our series was $90 \%$, which is comparable to the success rate reported in the literature for Chow's technique. A single-centre study was published in 2002 , to evaluate the results of endoscopic carpal tunnel release (ECTR) using the dual portal Chow technique in a large series of patients. A total of 2,675 procedures in 1,886 patients were performed during a thirteen-year period. Follow-up evaluation was performed in 2,402 (90\%) cases. The success rate was $95 \%$ and the recurrence rate was $0.5 \%$, suggesting that ECTR for carpal tunnel syndrome is a reliable procedure with a high success rate. Based on their thirteen years of experience, they believe that the technique is safe and iatrogenic complications can be avoided with meticulous surgical technique ${ }^{7}$.

Diminution of postoperative pain, early return to normal activities and work, and less scar tenderness are the major benefits of a successful ECTR. In this study, using the modified Chow's technique there was significant improvement in symptoms as early as two weeks, which was evident by, improvement in two-point discrimination, improvement in the VAS score, the high percentage $(90 \%)$ of negative Phalen test and Tinel sign, no pillar pain in the majority of cases $(80 \%)$, low incidence of scar tenderness $(6.7 \%)$, and a majority of cases $(80 \%)$ that had improved or were paraesthesia free at two weeks. Improved hand function was evident by, grip strength exceeding preoperative levels at three months, pinch strength exceeding preoperative levels by six months, and improved range of motion of the wrist joint.

The transverse carpal ligament has been shown to serve three functions; it anchors the thenar and hypothenar musculature; provides transverse stability to the carpus; and, acts as a pulley for the flexor tendons. Division of this ligament can alter the normal function of the wrist and contribute to postoperative complications such as pillar pain and grip weakness. Sectioning of the flexor retinaculum causes certain muscles to lose their anatomic attachments due to muscular pull shifted distally and radially or ulnarly. Using the open technique, muscle shortening may occurred in the superficial head of the flexor pollicis brevis (up to $25 \%$ relative to rest length), the ulnar part of the abductor pollicis brevis up to $20 \%$ (opposition and adduction), the opponens pollicis up to $20 \%$, and the opponens digiti minimi up to $10 \%$. This shortening causes a loss of muscle strength. Additionally, release of the transverse carpal ligament may disrupt the alignment and tracking of the piso-triquetral joint causing pain. This piso-triquetral tracking or alignment problem could be a possible aetiology of pillar pain ${ }^{8}$. In ECTR, the superficial fascia, which is palmar to the transverse carpal ligament (interthenar fascia) is usually left intact after dividing the flexor retinaculum with one pass of the knife causing less widening of the carpal arch. This may contribute to less pillar migration with an associated decrease in postsurgical loss of strength and pillar tenderness, resulting in an earlier return to work ${ }^{9}$.

In total there were two cases $(6.7 \%)$ that had recurrence in the present study. Their symptoms improved after undergoing open carpal tunnel release. In one case, the intraoperative findings indicated the presence of fibrous tissue and adhesions surrounding the median nerve. In the other patient, there was scarring around proximal portion of the transverse carpal ligament. If the carpal ligament were incompletely released in these cases, the patients would not have had any resolution of symptoms. As both of these patients had resolution of symptoms at two weeks followed by a return of symptoms from six weeks onwards, the possible cause may be due to scarring rather than incomplete division of the transverse carpal ligament.

The actual incidence of complications after ECTR is unknown. There have been several reports in the recent 
literature of major neurovascular injuries associated with ECTR although most of them are case reports or small series reports. The major reason for this complication is probably lack of experience with the technique. A common mistake is placement of the entry portal in a position that is too ulnar. The checkpoints described by James C.Y. Chow et al. are useful guidelines to estimate proper portal placement ${ }^{7}$. In this study, there was a low complication rate and no serious complications. We have to consider however, that the surgeon has extensive experience with this type of surgery and performs it on a regular basis. The lack of a control group and use of a non-randomized small sample were the weaknesses of the study. However, the study had the advantage of a prospective series of patients who had surgery performed by a single surgeon. The follow-up evaluation approached a period of one year and involved the majority of patients.

\section{CONCLUSION}

ECTR using the modified Chow's technique is a reproducible and safe method for the treatment of carpal tunnel syndrome. It is associated with low rates of perioperative and late complications. Most patients can expect resolution of symptoms by two weeks, allowing them to return to work early. 


\section{REFERENCES}

1. Trumble TE, Diao E, Abrams RA, Gilbert-Anderson MM. Single portal endoscopic carpal tunnel release compared with open release: a prospective, randomized trial. J Bone Joint Surg 2002; 84-A: 1107-5.

2. Louis DS, Greene TL, Noellert RC. Complications of carpal tunnel surgery. J Neurosurg 1985; 62: 352-6.

3. Brown RA, Gelberman RH, Seiler JG, Abrahamsson S-O, Weiland AJ, Urbaniak JR, et al. Carpal tunnel release: A prospective, randomized assessment of open and endoscopic methods. J Bone Joint Surg 1993; 75A: 1265-75.

4. Kerr CD, Gittins ME, Sybert DR. Endoscopic versus open carpal tunnel release: clinical results. Arthroscopy 1994; 10: $266-9$.

5. Palmer DH, Paulson JC, Lane-Larsen CL, Peulen VK, Olson JD. Endoscopic carpal tunnel release: a comparison of two techniques with open release. Arthroscopy 1993 ;9: 498-508.

6. Nagle DJ, Fischer TJ, Harris GD, Hastings H 11l, Osterman AL, Palmer AK. A multicenter prospective review of 640 endoscopic carpal tunnel releases using the transbursal and extrabursal Chow techniques. Arthroscopy 1996; 12: 139-43.

7. Chow JCY, Hantes ME. Endoscopic carpal tunnel release: thirteen years' experience with the Chow technique. J Hand Surgery 2002; 27A:1011-8.

8. Seradge H, Seradge E. Piso-triquetral pain syndrome after carpal tunnel release. J Hand Surgery 1989;14: 858 -62.

9. Viegas S.F, Pollard A, Kaminski K. Carpal arch alteration and related clinical Status after endoscopic carpal tunnel release. $J$ Hand Surgery 1992; 17: 1012-6. 